\title{
Navigating the wheel of glucose control: where are we now?
}

\begin{abstract}
Strict glucose control has been shown to reduce microvascular complications in Diabetes. Navigating the wheel of glucose control is not as simple as it may appear. We have relied upon estimation of blood glucose or the HbAlc which reflects an average control over the past 3 months. Other methods like Serum Fructosamine and 1, 5 Anhydroglucitol can also detect glucose control over a shorter period of time. However, the major limitation is that they cannot predict short term glucose fluctuations or recognise hypoglycaemia. Improvement in technology has led to introduction of various forms of continuous glucose monitoring. Real time continuous monitoring within a closed loop has led to the development of the dream reality, the artificial pancreas. In this review, we will discuss the above methods and their limitations.
\end{abstract}

Keywords: glucose monitoring, HbAlc, fructosamine, continuous glucose monitoring, ambulatory glucose profile

\author{
Volume 7 Issue I - 2019
}

\section{Sujit Bhattacharya}

Department of Endocrinology \& Faculty of Medicine, CK Birla Group of Hospitals, Director of Endocrinology, SCB Diabetes \& Hormone Research Foundation, India

Correspondence: Sujit Bhattacharya, Department of Endocrinology \& Faculty of Medicine, CK Birla Group of Hospitals, Director of Endocrinology, SCB Diabetes \& Hormone Research Foundation, Gajraj Signature, 5A Sadananda Road, Kolkata 700026, West Bengal, India, Tel +9l (33) 40063658, Fax +91 (33) 40063658, Email bhattacharyas.endo@gmail.com

Received: February 21, 2018 | Published: January 04, 2019
Abbreviations: CGMS, continuous glucose monitoring system; AGP, ambulatory glucose profile

\section{Introduction}

It is well established that intensive glucose control leads to improved incidence of micro vascular outcomes in both Type 1 and Type 2 Diabetes. ${ }^{1,2}$ Guidelines have already laid down the glycaemic targets to improve endpoints. However, the most important issue of concern is the increased incidence of hypoglycaemia as we try to achieve stricter goals. ${ }^{1,2}$ We routinely monitor capillary blood glucose and $\mathrm{HbA} 1 \mathrm{c}$ for glucose control while newer indices like estimated average glucose (eAG) and MAG (Mean Average glucose) has gained popularity. In certain circumstances, Fructosamine and 1, 5 AnhydroGlucitol can be used but it reflects glucose control over a very short period of time. Mean Amplitude of glycaemic excursion $(\mathrm{MAGE})^{3}$ and Glycaemic variability (GV) are looked at mostly in research settings and clinical relevance is still doubtful. ${ }^{4-28}$

Continuous glucose monitoring is helpful in certain circumstances especially where intensive monitoring is required and study daily variations over a timed interval. Recently, another simpler version of continuous monitoring can be done using an Ambulatory Glucose profile. The last two methods have the advantage of not only identifying the glucose peaks but also the troughs which are clinically important in routine care. The efficacy of these intensive regimens requires an accurate method to estimate the degree of glucose control which can be achieved.

\section{Discussion}

\section{Navigating the wheel of glucose control}

Comprehensive glucose monitoring is not as simple as it may appear. We have been gluco-centric and focussed on reaching the target. Though well recognized, we have ignored the troughs of glucose which could be equally if not more dangerous and lifethreatening and seriously impair the quality of life.

\section{The ideal technique should have the following characteristics:}

a. Optimal visualisation of hyperglycaemia: proper recognition of the target, identify daily peak and nadir, identify intraday glycaemic excursion following meals and other activities, identify day to day variation, measure mean glucose exposure and measure total glucose exposure over an extended period of time.

b. Recognition and prediction of hypoglycaemia.

c. Translation into clinical events and correlation with hard endpoints.

d. No alteration at any point in the natural history of Diabetes.

e. No alteration at any point of the natural history of micro/macro vascular complications

f. No alteration with other associated or co-morbid conditions.

g. Simple, safe, effective, economical.

h. Accurate, precise, reproducible.

\section{The indices for defining glycaemic control:}

i. The mean blood glucose concentration has often been measured in clinical trials as the mean of values obtained at point of care testing by glucometer. Capillary blood glucose measured before and 2 hour after meals and at $12 \mathrm{AM}$ or $3 \mathrm{AM}$ each day. This can be often pain staking for the patient and difficult to comply with over a long time especially in a busy schedule given the asymptomatic nature of the disease. For the HCP, it may be difficult to interpret because of ineligible entries or fake entries or deliberate omission of abnormal values. This can be replaced with or supplemented by the more simple measurement of A1C but often there can be significant disparity between the two as well.

ii. The degree to which blood glucose concentrations fluctuate 
within the same day can be formally measured as the mean amplitude of glycaemic excursions. ${ }^{3}$

iii. The degree to which blood glucose concentrations fluctuate from day to day can be formally measured as the mean of daily differences. ${ }^{5}$

iv. The mean blood glucose has received the greatest attention. However a given mean blood glucose value may be associated with different mean glycaemic excursions and mean daily differences The approach to improve blood glucose control will be different depending upon the degree of large fluctuations over and above the mean blood glucose (Figure 1).

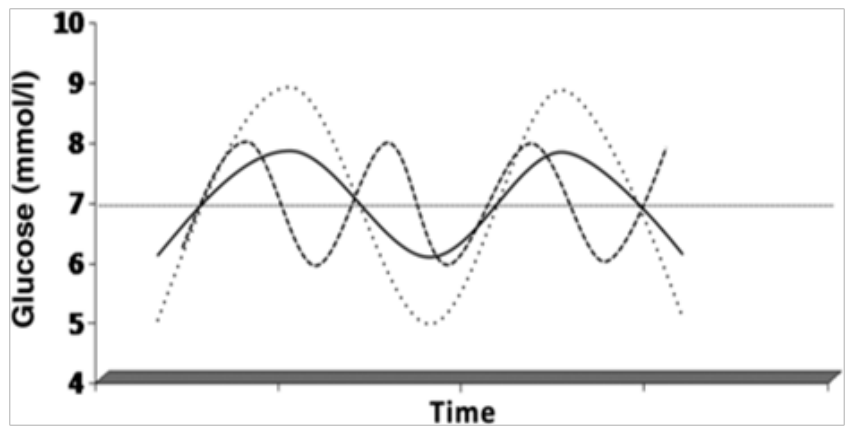

Figure I Visualization of glucose variability. Solid line: a given excursion.

Dashed line: higher glucose variability due to a higher frequency of oscillation. Dotted line: higher glucose variability due to larger amplitude.

Note that the mean and area under the curve are identical in the three situations.

\section{Estimation of blood glucose}

Glucose can attach to many proteins via a nonenzymatic, posttranslational process. ${ }^{6}$ This occurs in two stages.

a. A reversible reaction leads to the formation of an aldimine (or Schiff base)

b. This is followed by an Amadori rearrangement to form an irreversible ketoamine

The large majority of commercially available assays of glycated hemoglobin only measures the stable (irreversible) final product ketoamine and do not measure the labile (reversible) aldimine fraction. Therefore, they reflect long-term (two to three months) average glycemia, relatively unaffected by recent acute fluctuations in glucose levels. ${ }^{7,8}$

\section{Glycated haemoglobin}

The most widely used clinical test is measurement of glycated hemoglobin (also called A1C, hemoglobin A1C, glycohemoglobin, or $\mathrm{HbA1C}$ ). Red cells are freely permeable to glucose. As a result, glucose becomes irreversibly attached to hemoglobin at a rate dependent upon the prevailing glucose concentration. Approximately 1 percent of red cells are destroyed and a similar percentage replenished every day. Thus, the average amount of A1C changes in a dynamic way and indicates the mean blood glucose concentration over the lifespan of the red cell. ${ }^{7,8}$ Although the $\mathrm{A} 1 \mathrm{C}$ reflects mean blood glucose over the entire 120-day lifespan of the red blood cell, it correlates best with mean blood glucose over the previous 8 to 12 weeks. This strong correlation has been demonstrated in several studies including the
DCCT that have calculated average glucose on the basis of frequently measured, usually capillary, glucose levels. . $^{7,9,10}$

A large international study (A1C-Derived Average Glucose or ADAG study) calculated average glucose levels in appx 500 subjects (Type1, Type 2 and normal controls) using a combination of CGM and self-monitoring of blood glucose testing, and established a reliable regression equation that can be used to translate $\mathrm{A} 1 \mathrm{C}$ results into an estimated average glucose value. ${ }^{11}$

Studies suggest that A1C values may also be helpful in the diagnosis of impaired glucose tolerance or overt diabetes mellitus, being simpler to perform and to repeat than the oral glucose tolerance test.

Assay problems - In the past, the results of the DCCT could not be extrapolated widely, because of differences in methodology and a lack of standardization among laboratories. ${ }^{9}$ A new reference method has been established that will provide for even more reliable worldwide standardization of all A1C assays. ${ }^{12}$ With this new reference system, A1C results are reported globally in SI (Systeme International) units ( $\mathrm{mmol} / \mathrm{mol})$ and derived The National Glycohemoglobin Standardization Program (NGSP) units (the same values as reported currently as percent of total hemoglobin) using a master equation. An estimated average glucose (eAG), calculated from the $\mathrm{A} 1 \mathrm{C}$ result and based upon the results of the ADAG study described above, will be included in the report The eAG $(\mathrm{mg} / \mathrm{dL}$ or $\mathrm{mmol} / \mathrm{L}$ ) is a more relevant term for patients who self-monitor blood glucose. ${ }^{11}$ If there are differences in the eAG derived from the A1C result and the meter-calculated average glucose, further exploration is required. For example, if the eAG is higher than the patient's metercalculated average glucose, it is possible that fingerstick testing is not being performed at times when the blood glucose is highest, eg, after meals. If the eAG is lower than the meter average, a patient may be having undetected periods of low blood glucose, most often nocturnal hypoglycemia. In such cases, the timing of fingerstick blood glucose monitoring requires adjustment.

Sources of error-Although the international standardization of the $\mathrm{A} 1 \mathrm{C}$ assay has decreased potential technical errors in interpreting A1C results, there are other biological and patient-specific factors that may cause misleading results: ${ }^{13}$

1. A1C values are influenced by red cell survival. Thus, falsely high values in relation to a mean blood glucose values can be obtained when red cell turnover is low, resulting in a disproportionate number of older red cells. This problem can occur in patients with iron, vitamin B12, or folate deficiency anemia.

2. On the other hand, rapid red cell turnover leads to a greater proportion of younger red cells and falsely low A1C values. Examples include patients with hemolysis or anemia and those treated for iron, vitamin B12, or folate deficiency, and patients treated with erythropoietin..$^{14,15}$

3. Depending upon the methodology, the values may be falsely high in patients with abnormal hemoglobins (such as hemoglobin $\mathrm{F}[\mathrm{HbF}]$ ) or low with hemoglobin $\mathrm{S}(\mathrm{HbS}) .{ }^{16}$ However, many methods for measuring $\mathrm{A} 1 \mathrm{C}$ are no longer affected by the most common hemoglobin variants. The NGSP website contains current information about substances that interfere with A1C test results. 
4. A1C values may be falsely elevated or decreased in those with chronic kidney disease. False elevations may be due in part to analytical interference from carbamylated hemoglobin formed in the presence of elevated concentrations of urea, leading to false elevations in the $\mathrm{A} 1 \mathrm{C}$ level with some assays. False decreases in measured A1C may occur with hemodialysis and altered red cell turnover, especially in the setting of erythropoietin treatment.

Racial variation - Several studies have shown that A1C concentrations are higher in some ethnic groups (African American, Hispanic, Asian) than in white persons with similar plasma glucose concentrations. ${ }^{17,18}$ If differences in $\mathrm{A} 1 \mathrm{C}$ between races do exist, the differences appear to be small and have not been shown to significantly modify the association between $\mathrm{AlC}$ and cardiovascular outcomes, ${ }^{18}$ retinopathy, ${ }^{19,20}$ or nephropathy. ${ }^{21}$ In a cross-sectional study using the National Health and Nutrition Examination Survey (NHANES 2005 to 2008), the prevalence of retinopathy actually increased at a lower A1C level in blacks compared with whites (5.5 to 5.9 versus 6.0 to 6.4 percent). ${ }^{21}$ Potential mechanisms include genetic differences in intracellular glycation, glucose transport across the erythrocyte membrane, or in erythrocyte survival..$^{22,23}$

\section{Fructosamine}

Many proteins other than hemoglobin undergo nonenzymatic glycation, ${ }^{24}$ to form advanced glycosylation end products, which may play a direct role in the development of diabetic microvascular complications $^{25}$ Fructosamine is one ketoamine which can be measured in the laboratory. ${ }^{24}$ Some of the assays are cheaper and easier to perform than the $\mathrm{A} 1 \mathrm{C}$ assay and in general has a good correlation with $\mathrm{A} 1 \mathrm{C}$ values. ${ }^{25}$ There are, however, several potential problems with the use of serum fructosamine measurements:

1. The intraindividual variation for serum fructosamine is higher than that for $\mathrm{A} 1 \mathrm{C}$; so serum fructosamine concentrations must change more to be significant. ${ }^{26}$

2. The turnover of serum albumin is more rapid than that of hemoglobin ( 28 versus 120 days). So it reflect mean blood glucose values over a much shorter period of time (one to two weeks).

3. Serum fructosamine values must be adjusted if the serum albumin concentration is abnormal. ${ }^{27}$ Furthermore, false low values can occur in conditions like protein-losing enteropathy or nephrotic syndrome.

Intraday variations - The range over which blood glucose levels fluctuate vary within a day has been used to define a variety of "unstable diabetes" [ 4 and is a useful approximation of 5 to 7 point monitoring for several days. While unstable within-day glucose variation may increase the risk of hypoglycaemia, but there is no correlation with the development of microvascular complications. ${ }^{28}$

\section{I, 5-anhydroglucitol}

1, 5-anhydroglucitol (1,5-AG) is a naturally occurring dietary polyol which is normally filtered and completely reabsorbed by the kidneys leading to stable serum concentrations. However, renal reabsorption of 1,5-AG is competitively inhibited by glucose. Within 24 hours of a rise in serum glucose to beyond the renal threshold for glucose excretion i.e $>180 \mathrm{mg} / \mathrm{dL}(10 \mathrm{mmol} / \mathrm{L})$, serum $1,5-\mathrm{AG}$ concentrations fall as urinary losses increase. ${ }^{29-31}$ Thus, serum 1,5AG measurements reflect blood glucose values over the past 24 hours and provide information of daily glucose variations, whereas $\mathrm{A} 1 \mathrm{C}$ and fructosamine measurements reflect values over two to three months and one to two weeks, respectively.

Moreover, serum 1,5-AG may reflect postprandial glycaemic excursions better than A1C. ${ }^{31}$ However, this test is not readily available and neither there is any data to suggest that complementary measurement of 1,5-AG (GlycoMark assay) improves glycaemic control or complications of diabetes more than measurement of A1C alone. So, we do not typically measure $1,5-\mathrm{AG}$ concentrations and even with In patients who have high A1C values despite excellent premeal glucose levels, we prefer to test postprandial blood glucose one to two hours after meals to adjust medications.

Day to day variations-The extent to which blood glucose concentrations vary at the same time each day is another useful measure of overall glycaemic control. ${ }^{5}$ For patients who test their own blood glucose at the same times every day, this variability can be evaluated simply by scanning down columns of blood glucose measurements over several days. The usual explanation for large daily fluctuations is an erratic lifestyle in terms of eating or exercise habits. These problems should be corrected before increasing the insulin dose. The use of continuous glucose monitoring (CGM) devices can make the identification of within-day and between-day variation easier.

\section{Continuous glucose monitoring}

Continuous glucose monitoring (CGM) is a novel method for continuously monitoring blood glucose levels. The earlier CGM monitors did not provide real-time blood glucose data. These meters were downloaded in a clinic, where glucose trends were retrospectively analysed, evaluated and treatment decisions made. Real-time CGM (rt-CGM) systems provide immediate visual feedback about blood glucose levels to the patient. Both non-rt-CGM and rt-CGM methods provide visual graphs of blood glucose results over the period of time. In particular, CGM has successfully been used in clinical trials aimed at reducing $\mathrm{A} 1 \mathrm{C}$ in children and adults with type 1 diabetes in which the subjects could make immediate adjustments to insulin dosages. Few have included CGM as a method to improve glucose control in patients with type 2 diabetes (T2DM). ${ }^{32-38}$

In a study examining the clinical effectiveness of continuous glucose sensing, those with real-time access to the visual display had a reduction in time spent in low and high glucose numbers and increased the amount of time within their target blood glucose levels. ${ }^{32,38}$

There is growing support for use of CGM as a novel method for facilitating behaviour change in people with T2DMStudies suggests that non-rt-CGM and rt-CGM may be efficacious interventions to improve healthy behaviours and decrease $\mathrm{A} 1 \mathrm{C}$; however, the studies did not address the subjective experiences related to wearing the CGM. Most recently, it has been reported that it is important to consider the psychosocial factors associated with CGM..$^{38,39}$

Findings from another study suggest that use of CGM in women with T2DM is well tolerated and offers a vivid picture of blood glucose patterns that are ordinarily missed in routine diabetes management. Overall, the women were not overly burdened by the physical and logistical requirements associated with wearing the CGM. Most of the complaints were related to technological and personal aspects of CGM, which should be minimized with newer CGM technologies. For a small subset of the women, the experience elicited negative emotions diabetes emotional distress. ${ }^{32,38}$ 
In conclusion, continuous glucose monitoring offers patients with T2DM and their health care practitioners a new tool for assessing the effects of real-life events on blood glucose levels. Future research may determine that the use of CGM in the clinical setting may be more effective than routine use of SBGM at home for improving diabetes health outcomes (Figure 2).

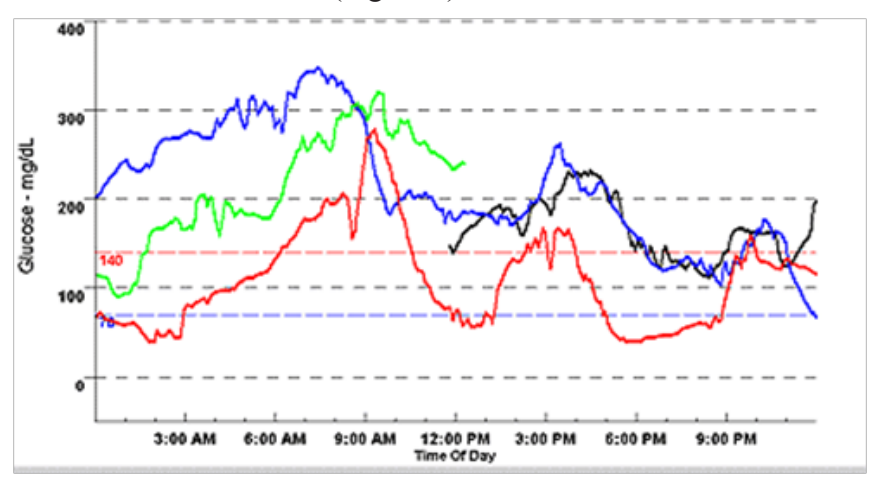

Figure 2 Continuous Glucose monitoring.

\section{Ambulatory glucose profile}

Current glucose monitoring tools have important shortcomings/ limitations. ${ }^{40-50}$

For the broad population of people living with diabetes, improved methods for assessing glucose values is needed.

HbA1c: Provides a 90-day average and considered the Standard of Care, but

i. Areas of hypoglycemia and hyperglycemia are unknown and not revealed. Glucose fluctuations throughout the day and night are unknown.

ii. SMBG Provides glucose information for only points in time, but

iii. Even when testing 7 times a day, can often miss hypoglycemia and hyperglycemia. Overnight data is impractical.

Inconsistency with testing blood glucose levels inadequate time to detect patterns in handwritten logbooks during short office visits. CGM: Provides a lot of glucose data, but

i. Reports can be difficult to interpret and gain insight.

ii. High start-up cost and only one patient per device; products are difficult to use, require cleaning \& disinfection, as well as detailed patient training.

AGP is constructed from a modal day plot, which collapses glucose data over several days or weeks. The data is plotted according to time as if they occurred over 24 hours. ${ }^{43,45-46,49-50}$ It is a visual snapshot of a patient's typical day, revealing patterns of:

1. Hypoglycemia

2. Hyperglycemia

3. Glycaemic Variability

The FreeStyle Libre Pro sensor has a sterile, thin flexible fibre that is inserted just under the skin to continuously measure glucose in the body using interstitial fluid (ISF). ${ }^{43-50}$

1. Wired enzyme based sensor is factory-calibrated to eliminate finger stick calibration
2. Wired Enzyme technology achieves stable sensor performance

a. Not dependent on oxygen to provide glucose readings

b. Sensor operates at a very low electrical potential.

Glucose readings-14 days vs. 30 days

Data suggest that a 12-15 day period of monitoring every 3 months may be needed to optimally assess overall glucose control. (Data on file. Abbott Laboratories Limited).

AGP reveals glucose variability by 2 measures.

The distance between the 10th and 90th percentile curves

A median curve that rises and falls shows glucose instability across the day (Figure 3).

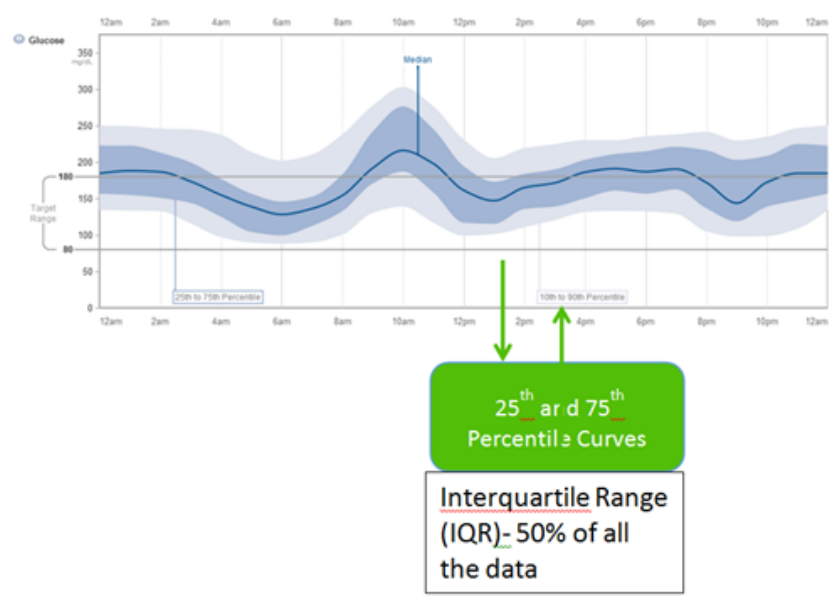

Figure 3 Ambulatory glucose profiles.

KEY benefits of AGP. ${ }^{44-46,49-50}$

HCP: The AGP graph clearly illustrates patterns in glucose levels. This enables HCPs to detect potential problems, such as hypoglycemia, hyperglycemia, and glycaemic variability (day to day) and glycaemic stability (intraday) more quickly.

GV helps us predict hypoglycemia: This amount of data is not available with SMBG even with the most engaged patient.

Patients: The information in the AGP graph is presented in a simplified way that facilitates understanding of glucose patterns.

The AGP graph provides a link between day-to-day behavior and glucose levels.

\section{Artificial pancreas:}

With the increasing availability of continuous glucose monitoring (CGM) and continuous subcutaneous insulin infusion (CSII), the artificial pancreas (AP) (the commonly accepted term for closed-loop control [CLC] of blood glucose [BG] levels in diabetes) has become a hot area in translational research and industrial development. After a prolonged period of inpatient, clinical research centre trials using cumbersome systems, the field has progressed rapidly over the past 2 years to long-term, free-living studies running AP algorithms on smartphones. Although it is still not a cure, the AP is the most promising advance in the treatment of diabetes at this time. ${ }^{42}$

Currently it is established that an Artificial Pancreas is a change in the treatment paradigm - not a single-function CGM or CSII 
device but an adaptable wearable network encompassing the patient in a digital treatment ecosystem. There is now a conviction that a mechanical solution to the problem of maintaining strict control of diabetes without increasing the risk of hypoglycaemia is rapidly progressing to a reality. ${ }^{51}$

\section{Conclusion}

Navigating the wheel of glucose control has never been easy in the dark, deep sea of complications in Diabetes especially in the murky waters of hypoglycaemia. Intensive glucose control has led us to look beyond blood glucose and focus on improved estimation \& standardisation of HBAlc. However the major limitation was identifying glucose fluctuations and specially recognising hypoglycaemia. Various forms of continuous glucose monitoring systems have been introduced over the past decade. It seems we are apparently heading towards the shore with the progress of science and digital technology. The dream reality of an Artificial Pancreas is a change in the treatment paradigm. The future holds in simplification of the complex technology and in translation into cost-effective clinical benefits with improved quality of life.

\section{Acknowledgments}

None.

\section{Conflict of interest}

The author declares there is no conflict of interest.

\section{References}

1. Nathan DM, Genuth S, Lachin J, et al. The effect of intensive treatment of diabetes on the development and progression of long-term complications in insulin-dependent diabetes mellitus. $N$ Engl $J$ Med. 1993;329(14):977-986.

2. King P, Peacock I, Donnelly R. The UK Prospective Diabetes Study (UKPDS): clinical and therapeutic implications for type 2 diabetes. $\mathrm{Br}$ J Clin Pharmacol. 1999;48(5):643-648.

3. Service FJ, Molnar GD, Rosevear JW, et al. Mean amplitude of glycaemic excursions, a measure of diabetic instability. Diabetes. 1970;19(9):644-655.

4. Hans DeVries J. Glucose Variability: Where It Is Important and How to Measure It. Diabete. 2013;62(5):1405-1408.

5. Molnar GD, Taylor WF, Ho MM. Day-to-day variation of continuously monitored glycaemia: a further measure of diabetic instability. Diabetologia. 1972;8(5):342-348.

6. Bunn HF, Haney DN, Gabbay KH, et al. Further identification of the nature and linkage of the carbohydrate in hemoglobin A1c. Biochem Biophys Res Commun. 1975; 67(1):103-109.

7. Nathan DM, Singer DE, Hurxthal K, et al. The clinical information value of the glycosylated hemoglobin assay. N Engl J Med. 1984;310(6):341346.

8. Goldstein DE. Is glycosylated hemoglobin clinically useful? $N$ Engl $J$ Med. 1984;310:384-385.

9. Rohlfing CL, Wiedmeyer HM, Little RR, et al. Defining the relationship between plasma glucose and $\mathrm{HbA}(1 \mathrm{c})$ : analysis of glucose profiles and $\mathrm{HbA}(1 \mathrm{c})$ in the Diabetes Control and Complications Trial. Diabetes Care. 2002;25(2):275-278.

10. Nathan DM, Turgeon H, Regan S. Relationship between glycated haemoglobin levels and mean glucose levels over time. Diabetologia. 2007;50(11):2239-2244.

11. Nathan DM, Kuenen J, Borg R, et al. Translating the A1C assay into estimated average glucose values. Diabetes Care. 2008;31(8):14731478 .

12. Hanas R, John G, International HBAlc Consensus Committee. 2010 consensus statement on the worldwide standardization of the hemoglobin A1C measurement. Diabetes Care. 2010;33:1903-1904.

13. National Glycohemoglobin Standardization Program (NGSP) website, which contains up to date information about substances that interfere with glycohemoglobin (HbAlc) test results.

14. Panzer S, Kronik G, Lechner K, et al. Glycosylated hemoglobins (GHb): an index of red cell survival. Blood. 1982;59(6):1348-1350.

15. Ng JM, Cooke M, Bhandari S, et al. The effect of iron and erythropoietin treatment on the $\mathrm{A} 1 \mathrm{C}$ of patients with diabetes and chronic kidney disease. Diabetes Care. 2010;33(11):2310-2313.

16. Roberts WL, Safar-Pour S, De BK, et al. Effects of hemoglobin C and $\mathrm{S}$ traits on glycohemoglobin measurements by eleven methods. Clin Chem. 2005;51(4):776.

17. Herman WH, Cohen RM. Racial and ethnic differences in the relationship between $\mathrm{HbAlc}$ and blood glucose: implications for the diagnosis of diabetes. J Clin Endocrinol Metab. 2012;97(4):1067-1072.

18. Bergenstal RM, Gal RL, Connor CG, et al. Racial Differences in the Relationship of Glucose Concentrations and Hemoglobin A1c Levels. Ann Intern Med. 2017;167(2):95-102.

19. Selvin E, Steffes MW, Zhu H, et al. Glycated hemoglobin, diabetes, and cardiovascular risk in nondiabetic adults. $N$ Engl $J$ Med. 2010;362(9):800-811.

20. Tsugawa Y, Mukamal KJ, Davis RB, et al. Should the hemoglobin A1c diagnostic cutoff differ between blacks and whites? A cross-sectional study. Ann Intern Med. 2012;157(3):153-159.

21. Selvin E, Ning Y, Steffes MW, et al. Glycated hemoglobin and the risk of kidney disease and retinopathy in adults with and without diabetes. Diabetes. 2011;60(1):298-305.

22. Cohen RM, Holmes YR, Chenier TC, et al. Discordance between $\mathrm{HbA} 1 \mathrm{c}$ and fructosamine: evidence for a glycosylation gap and its relation to diabetic nephropathy. Diabetes Care. 2003;26(1):163-167.

23. Cohen RM, Franco RS, Khera PK, et al. Red cell life span heterogeneity in hematologically normal people is sufficient to alter HbAlc. Blood. 2008;112(10):4284-4291.

24. Armbruster DA. Fructosamine: structure, analysis, and clinical usefulness. Clin Chem. 1987;33:2153-22163.

25. Vlassara H. Protein glycation in the kidney: role in diabetes and aging. Kidney Int. 1996;49(6):1795.

26. Narbonne H, Renacco E, Pradel V, et al. Can fructosamine be a surrogate for $\mathrm{HbA}(1 \mathrm{c})$ in evaluating the achievement of therapeutic goals in diabetes? Diabetes Metab. 2001;27(5 Pt 1):598.

27. Howey JE, Bennet WM, Browning MC, et al. Clinical utility of assays of glycosylated haemoglobin and serum fructosamine compared: use of data on biological variation. Diabet Med. 1989;6(9):793-796.

28. Kilpatrick ES, Rigby AS, Atkin SL. The effect of glucose variability on the risk of microvascular complications in type 1 diabetes. Diabetes Care. 2006; 29(7): 1486.

29. Buse JB, Freeman JL, Edelman SV, et al. Serum 1,5-anhydroglucitol (GlycoMark ): a short-term glycaemic marker. Diabetes Technol Ther: 2003;5(3):355-363. 
30. Dungan KM. 1,5-anhydroglucitol (GlycoMark) as a marker of shortterm glycaemic control and glycaemic excursions. Expert Rev Mol Diagn. 2008;8(1):9-19.

31. Stettler C, Stahl M, Allemann S, et al. Association of 1,5-anhydroglucitol and 2-h postprandial blood glucose in type 2 diabetic patients. Diabetes Care. 2008;31:1534.

32. Garg S, Zisser H, Schwartz S, et al. Improvement in glycaemic excursions with a transcutaneous, real-time continuous glucose sensor: a randomized controlled trial. Diabetes Care. 2006;29(1):44-50.

33. Deiss D, Bolinder J, Riveline JP, et al. Improved glycaemic control in poorly controlled patients with type 1 diabetes using real-time continuous glucose monitoring. Diabetes Care. 2006;29(12):2730 2732 .

34. JDRF CGM Study Group. JDRF randomized clinical trial to assess the efficacy of real-time continuous glucose monitoring in the management of type 1 diabetes: research design and methods. Diabetes Technol Ther. 2008;10(4):310-321.

35. Buckingham B, Xing D, Weinzimer S, et al. Use of the DirecNe applied treatment algorithm (DATA) for diabetes management with a real-time continuous glucose monitor (the FreeStyle navigator). Pediatr Diabetes. 2008;9(2):142-147.

36. Allen NA, Fain JA, Braun B, et al. Continuous glucose monitoring counseling improves physical activity behaviors of individuals with type 2 diabetes: a randomized clinical trial. Diabetes Res Clin Pract. 2008;80(3):371-379.

37. Yoo HJ, An HG, Park SY, et al. Use of a real time continuous glucose monitoring system as a motivational device for poorly controlled type 2 diabetes. Diabetes Res Clin Pract. 2008;82(1):73-79.

38. Ritholz M. Is continuous glucose monitoring for everyone? Consideration of psychosocial factors. Diabetes Spectrum. 2008;21(4):287-289.

39. Gilliam LK, Hirsch IB. Practical aspects of real-time continuous glucose monitoring. Diabetes Technol Ther. 2009;11(suppl 1):S75-S82.

40. Fritschi C, Quinn L, Sue Penckofer S, et al. Continuous glucose monitoring: the experience of women with type 2 diabetes. Diabetes Educ. 2010;36(2):250-257.

41. Block JM. Continuous glucose monitoring: changing diabetes behavior in real time and retrospectively. J Diabetes Sci Technol. 2008;2(3):484 489.

42. Seely EW. Current Opinion in Endocrinology, Diabetes \& Obesity. Editorial overview. Curr Opin Endocrinol Diabetes Obes. 2005;12(4):273-279.

43. Kovatchev B, Tamborlane WB, Cefalu WT, et al. The Artificial Pancreas in 2016: A Digital Treatment Ecosystem for Diabetes. Diabetes Care. 2016;39(7):1123-1126.

44. Mazze RS, Strock E, Wesley D, et al. Characterizing glucose exposure for individuals with normal glucose tolerance using continuous glucose monitoring and ambulatory glucose profile analysis. Diabetes Technol Ther. 2008;10(3):140-159.

45. Matthaei S, Dealaiz RA, Bosi E, et al. Consensus recommendations for the use of Ambulatory Glucose Profile in clinical practice. $\mathrm{Br} J$ Diabetes Vasc Dis. 2014;14:153-157.

46. Bergenstal RM, Ahmann AJ, Bailey T, et al. Recommendations for standardizing glucose reporting and analysis to optimize clinical decision making in diabetes: the Ambulatory Glucose Profile (AGP). Diabetes Technol Ther. 2013;15(3):198-211.

47. Serrano K. FDA supports standardized reporting and analysis on CGM devices. Diabetes Technol Ther. 2013;15(4):348.

48. Kaufman FR. It's time for a standard glucose report from CGM Diabetes Technol Ther. 2013;15(3):197.

49. Aaron JK, Sanjoy D. It's time to move from the A1c to better metrics for diabetes control. Diabetes Technol Ther. 2013;15(3):1946.

50. Matthaei S. Assessing the value of ambulatory glucose profile (AGP) in clinical practice. Br J Diabetes Vasc Dis. 2014;4:148-152.

51. Dunn TC, Crouther N. Assessment of the variance of the ambulatory glucose profile over 3 to 20 days of continuous glucose monitoring. Diabetologia. 2010;53(Suppl. 1):S421. 